\title{
Reinforcing Adherence to Lipid-lowering Therapy after an Acute Coronary Syndrome: A Pragmatic Randomized Controlled Trial
}

Stefanos Zafeiropoulos ( $\sim$ szafeirop@gmail.com )

Elmezzi Graduate School https://orcid.org/0000-0002-3284-2515

loannis Farmakis

AHEPA University Hospital

Anastasios Kartas

AHEPA University Hospital

Alexandra Arvanitaki

Konstantinos Arvanitakis

Vasileios Nevras

Areti Pagiantza

Stavros Zanos

George Giannakoulas

AHEPA University Hospital

\section{Research Article}

Keywords: Acute coronary syndromes, dyslipidemia, LDL-C goal, medication adherence

Posted Date: December 1st, 2020

DOI: https://doi.org/10.21203/rs.3.rs-118012/v2

License: (c) (1) This work is licensed under a Creative Commons Attribution 4.0 International License. Read Full License 


\section{Abstract}

Aims: Achieving the low-density lipoprotein cholesterol (LDL-C) goal following an acute coronary syndrome (ACS) is a milestone often missed due to suboptimal adherence to secondary prevention treatments. Whether improved adherence could result in reduced LDL-C levels is unclear. We aimed to evaluate an educational-motivational intervention to increase long-term lipid-lowering therapy (LLT) adherence and LDL-C goal attainment rate among post-ACS patients.

Methods: IDEAL-LDL was a parallel, two-arm, single-center, pragmatic, investigator-initiated randomized controlled trial. Hospitalized patients for ACS were randomized to a physician-led integrated intervention consisting of an educational session at baseline, followed by regular motivational interviewing phone sessions or usual care. Co-primary outcomes were the LLT adherence (measured by Proportion of Days Covered (PDC); good adherence defined as PDC $>80 \%)$, and LDL-C goal ( $<70 \mathrm{mg} / \mathrm{dl}$ or $50 \%$ reduction from baseline) achievement rate at one year.

Results: In total, 360 patients (mean age 62 years, 81\% male) were randomized. Overall, good adherence was positively associated with LDL-C goal achievement rate at one year. Median PDC was higher in the intervention group than the control group [0.92 (IQR, 0.82-1.00) vs. $0.86(0.62-0.98) ; p=0.03]$ while the intervention group had increased odds of good adherence (adjusted odds ratio: 1.76 (95\% confidence interval 1.02 to $2.62 ; \mathrm{p}=0.04)$. However, neither the rate of LDL-C goal achievement $(49.6 \%$ in the intervention vs. $44.9 \%$ in the control group; $p=0.49$ ) nor clinical outcomes differed significantly between the two groups.

Conclusion: A multifaceted intervention improved LLT adherence in post-ACS patients without a significant difference in LDL-C goal attainment. (IDEAL-LDL, ClinicalTrials.gov: NCT02927808)

\section{Introduction}

Worldwide, nearly 9 million people die from coronary artery disease (CAD) every year, and the years of lost life due to CAD keep increasing [1]. Meanwhile, acute coronary syndromes (ACS) are the life-threatening events in the natural history of CAD, and the risk of subsequent cardiovascular (CV) events rises beyond an ACS resulting in higher morbidity and mortality [2]. The pivotal role of LDL-C in secondary prevention post-ACS has been well studied and widely recognized [3]. Despite robust advances in the clinical management of ACS and dyslipidemia, patients face higher post-event mortality compared with the general population [4],[5], partially driven by recurrent CV events due to suboptimal control of risk factors [6].

Remarkable advances in lipid-lowering therapy (LLT) have been achieved, enhancing its therapeutic intensity potential [7]. However, the rate of achieving the low-density lipoprotein cholesterol (LDL-C) goal in a real-world setting is far from satisfactory and substantially lower among other traditional risk factors [8],[9]. Poor adherence to LLT remains a significant barrier to optimal care [10]. Nearly half of patients discontinue LLT [11],[12], resulting in an increased rate of recurrent cardiovascular events [13] and thus 
healthcare costs [14]. Although changing lifestyle behavior is complex and arduous, patient education and intensified care with regular doctor-patient communication have been proven efficient in reinforcing medication adherence [15]. Whether adherence-enhancing interventions could improve the LDL-C goal achievement rate remains unclear.

We aimed to determine whether an integrated intervention, comprised of structured patient education and motivational interviewing, could improve LLT adherence and LDL-C goal achievement rates compared with the standard of post-ACS care. Secondarily, we assessed the clinical outcomes, particularly death and a composite of major adverse cardiovascular events (MACE), and we identified prognostic factors for a subsequent MACE during the first year after the index event.

\section{Methods}

\section{Study design and setting}

The IDEAL-LDL (Motivational interviewing to support LDL-C therapeutic goals and lipid-lowering therapy compliance in patients with acute coronary syndromes) study was a parallel, two-arm, single-center, pragmatic, investigator-initiated randomized controlled trial. The study was conducted in AHEPA University Hospital, a high-volume center with over a million catchment populations. The adherenceenhancing intervention was developed based on the Theoretical Domains Framework and the Health Action Process Approach [16]. The trial has been registered in ClinicalTrials.gov (NCT02927808), approved by the Institutional Review Board of AHEPA Hospital and the Aristotle University Ethics Committee, and conducted according to the current version of the Declaration of Helsinki (2013). This study follows the Consolidated Standards of Reporting Trials (CONSORT) reporting guideline (the flowchart and checklist are presented in the Supplementary Materia). The full protocol of the study has already been published [17] and is illustrated schematically in Supplementary Material Figure S1. Briefly, the study enrolled consented patients admitted to our tertiary-care cardiology ward with a diagnosis of an ACS and discharged on LLT.

\section{Sample size and study population}

A sample size of 300 participants would provide at least $80 \%$ power (at a one-sided alpha level of 0.025 ) to detect an absolute increase of $15 \%$ in the LLT adherence rate (from 70\%) [18] and in the LDL-C goal achievement rate (from 20\%) [19] in the intervention group versus controls at one year. The inclusion criteria were patients aged $>18$ years with a documented diagnosis of ACS according to the Third and later Fourth Universal Definition of Myocardial Infarction [20]. The only clinically relevant exclusion criteria were contraindications to LLT, and any medical disorder that would compromise the completion of the study procedures.

\section{Baseline screening and randomization}


On the day of discharge, delegated investigators of the study assessed potentially eligible patients against the eligibility criteria (pre-screening), and if those were fulfilled, they continued to the screening. Data collection was performed in a standardized manner at the bedside on the same day. After successful screening, patients were randomized to one of the two treatment arms in a 1:1 ratio. An independent investigator performed the randomization procedure using computer-generated random numbers. Allocation concealment was ensured through sequentially numbered, opaque, sealed envelopes. Neither investigators nor patients were blinded to the intervention.

\section{Treatment groups}

In the control group, patients received the standard of care for an ACS by the healthcare personnel. Patients in this arm were contacted for an on-site or telemedicine follow-up visit at one year following the index event. Patients assigned in the intervention group received a proactive educational and motivational intervention, apart from the standard of care. In specific, at hospital discharge, patients had a 15-20 minute in-person discussion with the study personnel and were given a leaflet focusing on healthy lifestyle modifications, the value of high LLT adherence, and the clinical benefits of achieving the recommended LDL-C goal. Moreover, the intervention group was contacted for a 10-minute structured telephone interview at one month and six months after hospital discharge to be re-educated on the importance of LTT adherence and further motivated about the LDL-C goal. Patients were encouraged to contact the trained study personnel at any time for further relevant questions, but no medication change was performed in the context of the intervention. Like the control group, patients of the intervention arm were also scheduled for a 1-year follow-up visit for clinical and adherence assessment.

\section{Data sources}

All patients' data were recorded in structured electronic case report forms (eCRF) created exclusively for the IDEAL-LDL study. Each eCRF included contact details, baseline demographics (age, gender), somatometric parameters, complete medical and family history, cardiovascular risk factors, data regarding the index event, assigned in-hospital treatment, laboratory tests, medication, adherence, clinical, and safety events. We used the nationwide prescription register (www.e-prescription.gr), which hold individualized information, based on Social Security Number, of all claimed prescriptions from Greek pharmacies, to obtain adherence data and medication intake practices as well as the vital status of each subject. The rest clinical outcomes, apart from death, were collected either from our hospital records or through patient reports coupled with appropriate documentation that patients shared at the 1-year followup. Physicians blinded to patient randomization status reviewed and adjudicated all study relevant clinical and safety endpoints.

\section{Outcomes}


The study's primary endpoints were the adherence to LLT and the LDL-C goal achievement rate at 1year. Adherence was assessed by the Proportion of Days Covered (PDC) as recommended by the International Society for Pharmaceutical and Outcomes Research coupled with a self-report measure. PDC is calculated as the ratio of the number of days the medication covers the patient to the number of days the patient is eligible to have the medication on hand, namely 365 days in this trial [21]. Excess medication beyond one month after the assessment period was not included in the calculations. Good adherence was defined as PDC $>80 \%$, a cut-off that is widely used [22]. The LDL-C goal was defined as LDL-C $<70 \mathrm{mg} / \mathrm{dl}$ or at least a 50\% LDL-C reduction in accordance with the 2016 European Society of Cardiology (ESC)/European Atherosclerosis Society (EAS) guidelines [23]. A post-hoc analysis based on the 2019 ESC/EAS guideline-recommended goal (LDL-C $<55 \mathrm{mg} / \mathrm{dl}$ and at least a $50 \%$ reduction) was also performed [24]. Rest risk factors targets were based on the latest guidelines [25]. Specifically, the systolic blood pressure (SBP) target was defined at $120-130 \mathrm{mmHg}$ or at $130-140 \mathrm{mmHg}$ for patients aged $>65$ years. Body mass index (BMI) target was defined as $<25 \mathrm{~kg} / \mathrm{m}^{2}$, while regular exercise as at least 150 minutes of moderate aerobic activity or 75 minutes of vigorous aerobic activity a week, or a combination of them. The secondary endpoints included mean change from baseline values in LDL-C concentration at 1-year, all-cause mortality, and the classical three-point MACE (3P-MACE) composite, namely CV death, nonfatal MI, and nonfatal stroke as well as its individual components. Safety outcomes included all adverse events (AEs) focusing on the serious (SAEs) ones. Bleeding events were categorized or minor or major bleeding events. Detailed definitions of clinical outcomes are presented in the Supplementary Material.

\section{Statistical Analysis}

We performed the primary efficacy analysis based on the intention-to-treat population, which included all the participants who had been randomized. Continuous variables were tested for normality with the Shapiro-Wilk test and reported either as means \pm standard deviations (SD) or medians with interquartile ranges (IQRs). Student's t-test and Mann-Whitney $U$ test were performed for normally and non-normally distributed variables, respectively. Absolute and relative frequencies were used for categorical variables, and comparisons were made using Pearson's $\chi^{2}$ test. A 2-sided alpha level of 0.05 was used. Mortality rates with $95 \%$ confidence intervals (Cls) were estimated using the Kaplan-Meier method. Multivariate Cox proportional hazards regression was performed to identify predictors for subsequent MACE with clinical parameters and cardiac biomarkers as exploratory variables. Only variables showing a $\mathrm{p}$-value $<0.2$ in univariate models were introduced in the multivariate model. The multivariate models were tested for multicollinearity between covariates. All the analyses and figures were performed with the R statistical software (version 3.5.2) (R Foundation for Statistical Computing).

\section{Results}

\section{Population characteristics}


From June 24, 2016, through April 5, 2019, a total of 360 patients were enrolled in the study. Baseline characteristics and concomitant medication at discharge were balanced between the two groups, except for the proportion of beta-blocker prescription (Table 1). All patients were prescribed LLT; of those, 304 (84.4\%) were given a high-intensity statin. The loss to follow-up was 25 patients (7\%); 17 died, and 8 withdrew the informed consent.

At 1-year follow-up, out of 278 patients with an LDL-C measurement, 131 (47.1\%) had attained the LDL-C goal, whereas the respective number for the 2019 ESC/EAS Guidelines' stricter goal was 31 (11.2\%). Regarding other risk factors, $61.1 \%$ of the patients achieved the SBP target, $78.9 \%$ were non-smokers, $54.0 \%$ reported regular exercise, and $17.0 \%$ had BMI $<25 \mathrm{~kg} / \mathrm{m}^{2}$. Specifically, median LDL-C was 71.4 $\mathrm{mg} / \mathrm{dl}$ (IQR, 57.0-93.0), SBP was $125.0 \mathrm{mmHg}$ (IQR, 120.0-135.0) and BMI was $28.4 \mathrm{~kg} / \mathrm{m}^{2}$ (IQR, 26.030.9).

Table 1. Patients' characteristics at baseline grouped by treatment arm. 


\begin{tabular}{|c|c|c|c|c|}
\hline Characteristic & $\begin{array}{l}\text { Overall } \\
(n=360)\end{array}$ & $\begin{array}{l}\text { Control } \\
(n=180)\end{array}$ & $\begin{array}{l}\text { Intervention } \\
(\mathrm{n}=180)\end{array}$ & $\begin{array}{l}\mathrm{p}- \\
\text { value }\end{array}$ \\
\hline Age (years) & $\begin{array}{l}62.0(55.0- \\
73.0)\end{array}$ & $\begin{array}{l}63.0(54.8- \\
74.0)\end{array}$ & $\begin{array}{l}61.5(57.0- \\
70.3)\end{array}$ & 0.40 \\
\hline BMI $\left(\mathrm{kg} / \mathrm{m}^{2}\right)$ & $\begin{array}{l}27.5(25.3- \\
30.9)\end{array}$ & $\begin{array}{l}27.4(25.0- \\
30.5)\end{array}$ & $\begin{array}{l}27.8(25.7- \\
30.9)\end{array}$ & 0.31 \\
\hline Female sex, n (\%) & $67(19)$ & $33(18)$ & $34(19)$ & 0.89 \\
\hline \multicolumn{5}{|l|}{ Risk factors } \\
\hline Arterial hypertension, n (\%) & $194(54)$ & $102(57)$ & $92(51)$ & 0.20 \\
\hline Dyslipidemia, n (\%) & $135(38)$ & $60(33)$ & $75(41)$ & 0.12 \\
\hline Diabetes Mellitus, $\mathrm{n}(\%)$ & $105(29)$ & $52(29)$ & $53(29)$ & 0.91 \\
\hline Smoking, $\mathrm{n}(\%)$ & $186(52)$ & $90(50)$ & $96(53)$ & 0.52 \\
\hline Regular exercise, n (\%) & $143(40)$ & $70(39)$ & $73(41)$ & 0.75 \\
\hline \multicolumn{5}{|l|}{ History } \\
\hline Established CAD, n (\%) & $100(26)$ & $52(29)$ & $48(24)$ & 0.24 \\
\hline Previous MI, n (\%) & $81(23)$ & $42(23)$ & $39(21)$ & 0.52 \\
\hline PAD, n (\%) & $35(10)$ & $17(9)$ & $18(10)$ & 0.85 \\
\hline Stroke, n (\%) & $25(7)$ & $12(6)$ & $13(7)$ & 0.76 \\
\hline Heart failure, $n(\%)$ & $58(16)$ & $27(15)$ & $31(17)$ & 0.60 \\
\hline Family history of CAD, $n$ (\%) & $158(44)$ & $78(43)$ & $80(44)$ & 0.83 \\
\hline \multicolumn{5}{|l|}{ Type of event } \\
\hline STEMI, n (\%) & $199(55)$ & $104(58)$ & $95(53)$ & 0.42 \\
\hline NSTEMI, n (\%) & $116(32)$ & $53(30)$ & $63(34)$ & 0.33 \\
\hline Unstable angina, n (\%) & $45(13)$ & $23(13)$ & $22(12)$ & 0.86 \\
\hline \multicolumn{5}{|l|}{ Cholesterol (mg/dl) } \\
\hline Total & $171(145-202)$ & $170(145-201)$ & $171(146-202)$ & 0.69 \\
\hline Triglycerides & $130(95-178)$ & $130(94-180)$ & $130(96-177)$ & 0.90 \\
\hline HDL & $38(32-46)$ & $37(30-46)$ & $38(30-46)$ & 0.30 \\
\hline LDL & $102(79-128)$ & $102(79-124)$ & $104(80-128)$ & 0.68 \\
\hline Non-HDL & $132(103-160)$ & $132(103-162)$ & $132(104-159)$ & 0.95 \\
\hline eGFR $\left(\mathrm{mL} \cdot \mathrm{min}^{-1} \cdot 1.73 \mathrm{~m}^{2}\right)^{1}$ & $83(66-96)$ & $82(65-94)$ & $85(69-97)$ & 0.25 \\
\hline
\end{tabular}




\begin{tabular}{|c|c|c|c|c|}
\hline $\begin{array}{l}\text { High-sensitivity troponin (max) } \\
\text { (ng/l) }\end{array}$ & $\begin{array}{l}1236(248- \\
3686)\end{array}$ & $\begin{array}{l}1266(241- \\
3764)\end{array}$ & $\begin{array}{l}1172(284- \\
3491)\end{array}$ & 0.69 \\
\hline NT-proBNP (pg/mL) & $\begin{array}{l}899(344- \\
2324)\end{array}$ & $\begin{array}{l}800(344- \\
2213)\end{array}$ & $\begin{array}{l}1106(355- \\
2613)\end{array}$ & 0.49 \\
\hline LVEF at discharge, $n(\%)$ & $50(43-55)$ & $50(42-55)$ & $50(45-55)$ & 0.28 \\
\hline Primary PCl, n (\%) & $289(81)$ & $143(81)$ & $146(82)$ & 0.68 \\
\hline Conservative treatment, $\mathrm{n}(\%)$ & $71(19)$ & $36(20)$ & $34(18)$ & 0.64 \\
\hline Total length of hospitalization & $8.0(6.8-10.0)$ & $8.0(7.0-10.0)$ & $7.0(6.0-9.0)$ & 0.06 \\
\hline \multicolumn{5}{|l|}{ Medication at discharge } \\
\hline Statin, n (\%) & $351(98)$ & $174(97)$ & $177(98)$ & 0.68 \\
\hline High intensity, n (\%) & $304(84)$ & $150(83)$ & $154(85)$ & 0.58 \\
\hline Ezetimibe, n (\%) & $39(11)$ & $20(11)$ & $19(11)$ & 0.96 \\
\hline Antiplatelet, n (\%) & $359(100)$ & $179(100)$ & $180(100)$ & 0.85 \\
\hline ACE inhibitor, $n$ (\%) & $160(46)$ & $83(47)$ & $77(44)$ & 0.58 \\
\hline ARB, n (\%) & $58(16)$ & $30(17)$ & $28(16)$ & 0.70 \\
\hline Beta-blocker, n (\%) & $309(89)$ & $146(85)$ & $163(93)$ & $0.03^{*}$ \\
\hline
\end{tabular}

BMI, Body Mass Index; CAD, Coronary artery disease; MI, Myocardial infarction; PAD, Peripheral arterial disease; STEMI, ST-elevation Ml; NSTEMI, non-ST-elevation Ml; HDL, High-density cholesterol, LDL, Lowdensity cholesterol; eGFR, estimated glomerular filtration rate; NT-proBNP, N-terminal pro-b-type natriuretic peptide; LVEF, left ventricular ejection fraction; $\mathrm{PCl}$, Percutaneous coronary intervention; ARB, Angiotensin II receptor blocker; ACE, Angiotensin-converting enzyme; Data were reported as absolute frequencies for discrete variables and medians (IQR) for continuous variables. ${ }^{1}$ (CKD-EPI)

\section{Adherence and LDL-C goal achievement rates}

At 1-year follow-up, the median PDC was 0.92 (IQR, 0.82-1.00) in the intervention group and 0.86 (0.620.98 ) in the control group ( $p=0.03$ ) (Figure $1 A)$. In the intervention group, 126 out of 167 patients $(75.5 \%)$ had good adherence compared with 111 out of 168 patients $(66.1 \%)$ in the control group (adjusted odds ratio $1.76,95 \% \mathrm{Cl} 1.02$ to $2.62 ; \mathrm{p}=0.04$ ). However, the LDL-C goal achievement rates did not differ between the two groups neither according to the 2016 Guidelines (49.6\% in the intervention group vs. $44.9 \%$ in the controls; $p=0.49)$ (Figure 1B) nor according to the 2019 ESC Guidelines (10.1\% vs. $12.3 \% ; p=0.76)$.

Median LDL-C values were similar between the intervention and the control group at 1-year follow-up [70 $\mathrm{mg} / \mathrm{dl}(\mathrm{IQR}, 56.4-93.0)$ vs. $73 \mathrm{mg} / \mathrm{dl}(58.0-92.8) ; \mathrm{p}=0.55]$. No significant differences between the two arms were found for the mean change from baseline LDL-C and for all other cholesterol values (Table 2). 
Achievement rates for other risk factors, namely smoking cessation, SBP, and BMI were also similar among the two groups (Supplementary material Table S2). Besides, self-reported medication adherence was similar between the two groups (mean $97 \%$ in the intervention vs. $96 \%$ in the control group; $p=0.73$ ).

Table 2. Goal achievements and laboratory values of the patients at 1-year follow-up.

\begin{tabular}{|llll|}
\hline Characteristic & $\begin{array}{l}\text { Control } \\
(\mathrm{n}=138)\end{array}$ & $\begin{array}{l}\text { Intervention } \\
(\mathrm{n}=140)\end{array}$ & p-value \\
\hline LDL-C (mg/dl) & $73(58.0-92.8)$ & $70(56.4-93.0)$ & 0.55 \\
\hline Mean LDL-C change (mg/dl) & $-30.2 \pm 42.6$ & $-27.5 \pm 36.7$ & 0.57 \\
\hline HDL-C (mg/dl) & $41.5(35.0-49.3)$ & $42.5(35.0-52.0)$ & 0.37 \\
\hline Non-HDL-C (mg/dl) & $98.0(81.8-116.3)$ & $92.0(80.0-117.0)$ & 0.56 \\
\hline Triglycerides (mg/dl) & $112.5(88.5-145.5)$ & $121.0(88.0-163.5)$ & 0.33 \\
\hline Total cholesterol (mg/dl) & $141.0(126.0-159.0)$ & $139.0(125.0-169.0)$ & 0.95 \\
\hline SBP $(\mathbf{m m H g})$ & $126.0(121.0-136.0)$ & $124.0(119.0-134.0)$ & 0.72 \\
\hline
\end{tabular}

LDL, Low-density lipoprotein; C, Cholesterol; HDL, High-density lipoprotein, SBP, Systolic blood pressure; Data were reported as absolute frequencies for discrete variables and means $\pm S D$ or medians (IQR) for continuous variable

In our total population, patients with good adherence had greater mean LDL-C change from baseline [32.8 $\pm 40.4 \mathrm{mg} / \mathrm{dl}$ vs. $19.8 \pm 38.1 \mathrm{mg} / \mathrm{dl} ; \mathrm{p}=0.02$ ], lower LDL-C values [median $69.0 \mathrm{mg} / \mathrm{dl}($ IQR, 55.5-88.5) vs. $80.0 \mathrm{mg} / \mathrm{dl}(64.0-109.0) ; \mathrm{p}=0.004$ ] (Figure $2 \mathrm{~A}$ ) than those with poor adherence and were more likely to achieve the LDL-C goal (adjusted odds ratio $1.91,95 \% \mathrm{Cl} 1.09$ to $3.41 ; \mathrm{p}=0.02$ ). Figure $2 \mathrm{~B}$ shows that adherence was inversely related to mean LDL-C change from baseline for both experimental arms. Concerning the LLT at 1-year follow-up, 324 patients (96.7\%) were under LLT, particularly, 323 (96.4\%) were treated with statins; of those, 234 (72.4\%) were on a high-intensity statin while 57 (17.6\%) were taking a combination with ezetimibe.

\section{Clinical outcomes}

Our cohort's cumulative incidence of all-cause mortality was $4.8 \%(95 \% \mathrm{Cl} 2.5$ to 6.9$)$, and 3P-MACE was $11.2 \%$ (95\% $\mathrm{Cl} 8.0$ to 14.8 ) at one year following the index ACS event. In total, 37 patients $(11.2 \%)$ experienced a 3P-MACE; CV death occurred in 12 patients (3.7\%), nonfatal MI in 24 (7.3\%), non-fatal 
stroke in $1(0.3 \%)$. Besides, seven patients $(2.1 \%)$ were hospitalized for heart failure. Kaplan-Meier analyses of death from any cause and 3P-MACE grouped by assigned arm are demonstrated in Figure 3. Event rates for all-cause mortality and the composite outcome were similar between the two arms [hazard ratio (HR), $1.13 ; 95 \% \mathrm{Cl} 0.4$ to $2.9 ; \mathrm{p}=0.8$ and $\mathrm{HR}, 1.1 ; 95 \% \mathrm{Cl} 0.5$ to $1.7 ; \mathrm{p}=0.8$, respectively]. Concerning the safety outcomes, two patients had major bleeding (one in the intervention and one in the control group). Of the patients receiving LLT, six patients (1.8\%) complained of myalgias, and three of them discontinued statin therapy. No significant difference in safety outcomes was observed between the two groups.

In the univariate analysis for 3P-MACE predictors, age, history of arterial hypertension, polyvascular disease, established CAD before the index event, and eGFR $<30 \mathrm{ml} / \mathrm{min} / 1.73 \mathrm{~m}^{2}$ at baseline had prognostic value for a subsequent 3P-MACE during the first year. Of note, in the multivariate analysis, only the history of arterial hypertension remained a statistically significant predictor, as shown in Table 3 .

Table 3. Risk factors and goal achievement related to 3P-MACE.

\begin{tabular}{|c|c|c|c|c|}
\hline \multirow[t]{2}{*}{ Risk factors } & \multicolumn{2}{|l|}{ Univariate analysis } & \multicolumn{2}{|l|}{ Multivariate analysis } \\
\hline & OR $(95 \% \mathrm{Cl})$ & P-Value & Adjusted OR (95\% Cl) & P-Value \\
\hline $\mathrm{Age}^{1}$ & $1.02(0.99-1.05)$ & 0.13 & $1.02(0.97-1.03)$ & 0.92 \\
\hline Female & $1.24(0.81-2.72)$ & 0.59 & & \\
\hline Established CAD & $1.94(1.01-3.71)$ & $0.04^{*}$ & $1.37(0.69-2.74)$ & 0.37 \\
\hline Polyvascular disease & $2.08(1.00-4.29)$ & $0.04^{*}$ & $1.48(0.68-3.21)$ & 0.32 \\
\hline Arterial Hypertension & $2.93(1.34-6.42)$ & $0.007 * \star$ & $2.48(1.07-5.77)$ & $0.03 *$ \\
\hline Diabetes & $1.26(0.63-2.50)$ & 0.5 & & \\
\hline eGFR $<30 \mathrm{ml} / \mathrm{min} / 1.73 \mathrm{~m}^{2}$ & $3.36(1.19-9.50)$ & $0.02^{\star}$ & $5.49(1.80-16.69)$ & 0.16 \\
\hline Increased NT-proBNP2 & $1.62(0.49-5.3)$ & 0.40 & & \\
\hline LDL-C $<70 \mathrm{mg} / \mathrm{dl}$ & $1.43(0.57-3.57)$ & 0.43 & & \\
\hline
\end{tabular}

OR, Odds ratio; Cl; Confidence interval; $\mathrm{CAD}$, Coronary artery disease; eGFR, estimated glomerular filtration rate; NT-proBNP, N-terminal pro-b-type natriuretic peptide; LDL-C, Low-density lipoprotein cholesterol;

${ }^{7}$ continuous variable; 
${ }^{2} \mathrm{Age}>75$ and nt-proBNP $>450 \mathrm{pg} / \mathrm{ml}$ or age $<75$ and nt-proBNP $>125 \mathrm{pg} / \mathrm{ml}$

\section{Discussion}

In the era of patient-centered health care and more than ever demanding LDL-C goals, the IDEAL-LDL trial investigated the impact of a physician-led intervention with educational and motivational components in the LLT adherence and LDL-C goal achievement rate. The intervention group had higher odds of being adherent than the control group. No difference was observed in the achievement rates among the two experimental groups, although good adherence was positively associated with LDL-C goal achievement in the overall study population. In addition, clinical and safety endpoints were similar between the groups.

It is well established that increased adherence to secondary prevention medication, especially LLT, is associated with reduced MACE and all-cause mortality risk [13],[26]. However, up to 50\% of post-ACS patients discontinue LLT therapy over the first year [12]. A plethora of strategies [18],[27],[28] have been proposed to improve medication-taking behavior, and particularly, personalized, patient-focused programs involving regular contact with health care professionals have shown consistent efficacy in improving adherence and, thus, were the basis of our intervention. Notably, two pertinent meta-analyses of adherence-enhancing interventions in CAD patients confirmed a benefit in adherence [15],[29].

Nevertheless, most of the trials included in these meta-analyses did not report on treatment goals achievement and clinical outcomes and, as a result, the clinical benefit of increased adherence has not been thoroughly addressed. Our study failed to show an association of adherence-enhancing intervention and LDL-C between the intervention and control groups. In line with our findings, a recent adherenceenhancing intervention involving financial incentives showed increased adherence but similar LDL-C values among the experimental groups [30]. These results underline the importance of directly measuring clinical outcomes, rather than mere adherence, in trials with behavioral interventions. It must be acknowledged, however, that poor LDL-C goal attainment is to a large extent due to physician inertia rather than suboptimal patient adherence.

The 2016 ESC/EAS LDL-C goal is a cornerstone in patients' secondary prevention management, but its achievement rate remains suboptimal across Europe despite the upward trend. The EUROASPIRE IV [19] reported an achievement rate of $19.5 \%$, which climbed to $29 \%$ in EUROASPIRE V [31]. The latest LLT dedicated study -the DA VINCl study [32]- in 18 European countries demonstrated 2016 ESC/EAS goal attainment rate of $39 \%$, even greater than the abovementioned registries. However, the gaps between clinical guidelines and clinical practice for dyslipidemia management persist and will be further exacerbated by the stricter 2019 ESC/EAS guidelines. Specifically, in the DA VINCI study, only $18 \%$ of the secondary prevention patients achieved the 2019 ESC/EAS goals. In our population, nearly half of the patients achieved the 2016 LDL-C goal and $11.3 \%$ the 2019 LDL-C goal; these already remarkably high rates could explain our intervention's failure to achieve a greater attainment rate. 
Concerning LLT, the EUROASPIRE IV [19], V [31], and DA VINCI [32] studies demonstrated a significant down-titration of statin's intensity over the first year after the index event resulting in less than half of the patients treated with high-intensity statins at one year. The same downward trend in high-intensity utilization was also observed in IDEAL-LDL (high-intensity statin: $84.4 \%$ at discharge vs. $72.4 \%$ at 1-year). At 1-year follow-up, we reported a higher prescription rate of statins compared with EUROASPIRE IV [19] (86.0\%) and V [31] (84\%), and Rapsomaniki et al. [5] (varying from $76.1 \%$ to $86.7 \%)$. The higher use of statins in our population could be attributed to the inclusion criteria and our study's intervention and could partially justify the substantially increased LDL-C goal achievement rate. Besides, our population's mortality rate was low but similar to recent studies [4], probably attributed to the advances in revascularization and antithrombotic therapy that may have shifted the recurrent events after a year postindex ACS. Therefore, the small numbers of participants and clinical events prevent us from drawing firm conclusions.

Our study could have important clinical implications. It represents a real-world population of hospitalized patients with ACS and can provide insight into the practical implementation of the guidelinerecommended targets of ACS patients, thereby raising awareness of a patient group requiring intense therapy and close follow-up. The long-term prognostic value related to adherence may have clinical applications and be incorporated into CV prognostic scores. Adherence indices could be used to identify high-risk populations prone to medication interruption or discontinuation, which would benefit from multifaced interventions, including mobile applications [33] and biodegradable, ingestible sensors [34]. Secondary prevention through comprehensive cardiac rehabilitation has proven efficacy and costeffectiveness across a broad spectrum of CV disease [35]. In particular, a comprehensive, reinforced, educational, and behavioral intervention in the context of a rehabilitation program could reduce the risk of adverse CV events [36]. The adherence-enhancing interventions could also be cost-effective [37], delivered in this context, and established as part of routine care [35]. However, the use of rehabilitation programs varied significantly worldwide [38], and internationally accepted standards should be determined.

\section{Strengths and limitations}

One major limitation of our study is the small sample size; however, it is one of the largest among adherence-reinforcing studies with post-ACS patients. Approximately $21 \%$ of participants did not have a 12-month lipid panel test, despite investigators' efforts to urge participants to perform the test. In addition, not all of the patients with an ACS in our hospital have been included in this study, yet the majority of them, and, thus, selection bias cannot be excluded. Besides, the follow-up period was reasonable for adherence and LDL-C goal endpoints but perhaps too short for assessing clinical outcomes. Moreover, PDC is simple, objective, and currently, the most commonly used measure of medication adherence based on pharmacy data [39]. Therefore, it was preferred over self-report, which was the initially chosen measure, since those two have proven discordant [40], although new self-reported measures of adherence are emerging[41]. However, other direct adherence assessment methods, such as periodic count of unused drugs, pill count, could be more accurate. In addition, female sex and other 
races - apart from Caucasian - were under-represented in our population and may affect the

extrapolation of our findings. Lastly, this study, being single-center, has the advantage of the homogeneity in provided healthcare, integrity, and homogeneity in patients' data and laboratory values. However, it may not be representative of routine clinical practice across other national healthcare centers, especially in non-academic settings.

\section{Conclusions}

In patients being discharged from hospital after an ACS, a motivational-educational intervention, compared to the standard of care, led to improved adherence to LLT at one year. No improvement in the rate of LDL-C goal attainment or clinical outcomes was observed. Larger and longer trials are needed to investigate if the improved adherence is translated into clinical benefit.

\section{References}

1. Roth GA, Abate D, Abate $\mathrm{KH}$, et al.(2018) Global, regional, and national age-sex-specific mortality for 282 causes of death in 195 countries and territories, 1980-2017: a systematic analysis for the Global Burden of Disease Study 2017. Lancet. 392(10159):1736-1788. doi:10.1016/S01406736(18)32203-7

2. Jernberg T, Hasvold P, Henriksson M, Hjelm H, Thuresson M, Janzon M.(2015) Cardiovascular risk in post-myocardial infarction patients: Nationwide real world data demonstrate the importance of a long-term perspective. Eur Heart J. 36(19):1163-1170a. doi:10.1093/eurheartj/ehu505

3. Libby P, Buring JE, Badimon L, et al.(2019) Atherosclerosis. Nat Rev Dis Prim. 5(1):1-18. doi:10.1038/s41572-019-0106-z

4. Rossello X, Bueno H, Pocock SJ, et al.(2019) Predictors of all-cause mortality and ischemic events within and beyond 1 year after an acute coronary syndrome: Results from the EPICOR registry. Clin Cardiol. 42(1):111-119. doi:10.1002/clc.23116

5. Rapsomaniki E, Thuresson M, Yang E, et al.(2016) Using big data from health records from four countries to evaluate chronic disease outcomes: A study in 114364 survivors ofmyocardial infarction. Eur Hear J - Qual Care Clin Outcomes. 2(3):172-183. doi:10.1093/ehjqcco/qcw004

6. Zafeiropoulos S, Farmakis I, Kartas A, et al.(2020) Risk for Recurrent Cardiovascular Events and Expected Risk Reduction With Optimal Treatment 1 Year After an Acute Coronary Syndrome. Am J Cardiol. 133:7-14. doi:10.1016/j.amjcard.2020.07.046

7. Rosenson RS, Hegele RA, Fazio S, Cannon CP.(2018) The Evolving Future of PCSK9 Inhibitors. J Am Coll Cardiol. 72(3):314-329. doi:10.1016/j.jacc.2018.04.054

8. Kotseva K, De Backer G, De Bacquer D, et al.(2019) Lifestyle and impact on cardiovascular risk factor control in coronary patients across 27 countries: Results from the European Society of Cardiology ESC-EORP EUROASPIRE V registry. Eur J Prev Cardiol. 26(8):824-835.

doi:10.1177/2047487318825350

Page $13 / 18$ 
9. Farmakis I, Zafeiropoulos S, Pagiantza A, et al.(2019) Low-density lipoprotein cholesterol target value attainment based on 2019 ESC/EAS guidelines and lipid-lowering therapy titration for patients with acute coronary syndrome [published online ahead of print, 2020 Dec 9]. Eur J Prev Cardiol. Published online 2019. doi:10.1177/2047487319891780

10. De Vera MA, Bhole V, Burns LC, Lacaille D.(2014) Impact of statin adherence on cardiovascular disease and mortality outcomes: a systematic review. Br J Clin Pharmacol. 78(4):684-698. doi:10.1111/bcp.12339

11. Schwalm JD, Ivers NM, Natarajan MK, et al.(2015) Cluster randomized controlled trial of Delayed Educational Reminders for Long-term Medication Adherence in ST-Elevation Myocardial Infarction (DERLA-STEMI). Am Heart J. 170(5):903-913. doi:10.1016/j.ahj.2015.08.014

12. Hamood H, Hamood R, Green MS, Almog R.(2015) Determinants of adherence to evidence-based therapy after acute myocardial infarction. Eur J Prev Cardiol. 23(9):975-985. doi:10.1177/2047487315597209

13. Rasmussen JN, Chong A, Alter DA.(2007) Relationship between adherence to evidence-based pharmacotherapy and long-term mortality after acute myocardial infarction. J Am Med Assoc. 297(2):177-186. doi:10.1001/jama.297.2.177

14. Choudhry NK, Avorn J, Glynn RJ, et al.(2011) Full coverage for preventive medications after myocardial infarction. N Engl J Med. 365(22):2088-2097. doi:10.1056/NEJMsa1107913

15. Santo K, Kirkendall S, Laba TL, et al.(2016) Interventions to improve medication adherence in coronary disease patients: A systematic review and meta-analysis of randomised controlled trials. Eur J Prev Cardiol. 23(10):1065-1076. doi:10.1177/2047487316638501

16. Atkins L, Francis J, Islam R, et al.(2017) A guide to using the Theoretical Domains Framework of behaviour change to investigate implementation problems. Implement Sci. 12(1):77. doi:10.1186/s13012-017-0605-9

17. Boulmpou A, Kartas A, Farmakis I, et al.(2019) Motivational interviewing to support LDL-C therapeutic goals and lipid-lowering therapy compliance in patients with acute coronary syndromes (IDEAL-LDL) study: rationale and design. Hell J Cardiol. 60(4):249-253. doi:10.1016/j.hjc.2018.10.002

18. Vonbank A, Agewall S, Per Kjeldsen K, et al.(2017) Comprehensive efforts to increase adherence to statin therapy. Eur Heart J. 38(32):2473-2477. doi:10.1093/eurheartj/ehw628

19. Reiner, De Backer G, Fras Z, et al.(2016) Lipid lowering drug therapy in patients with coronary heart disease from 24 European countries - Findings from the EUROASPIRE IV survey. Atherosclerosis. 246:243-250. doi:10.1016/j.atherosclerosis.2016.01.018

20. Thygesen K, Alpert JS, Jaffe AS, et al.(2019) Fourth universal definition of myocardial infarction (2018). Eur Heart J. 40(3):237-269. doi:10.1093/eurheartj/ehy462

21. Peterson AM, Nau DP, Cramer JA, Benner J, Gwadry-Sridhar F, Nichol M.(2007) A checklist for medication compliance and persistence studies using retrospective databases. Value Heal. 10(1):312. doi:10.1111/j.1524-4733.2006.00139.x

Page $14 / 18$ 
22. Karve S, Cleves MA, Helm M, Hudson TJ, West DS, Martin BC.(2009) Good and poor adherence: Optimal cut-point for adherence measures using administrative claims data. Curr Med Res Opin. 25(9):2303-2310. doi:10.1185/03007990903126833

23. Catapano AL, Graham I, De Backer G, et al.(2016) 2016 ESC/EAS Guidelines for the Management of Dyslipidaemias. Eur Heart J. 37(39):2999-3058l. doi:10.1093/eurheartj/ehw272

24. Mach F, Baigent C, Catapano AL, et al.(2020) 2019 ESC/EAS Guidelines for the management of dyslipidaemias: lipid modification to reduce cardiovascular risk. Eur Heart J. 41(1):111-188. doi:10.1093/eurheartj/ehz455

25. Knuuti J, Wijns W, Achenbach S, et al.(2020) 2019 ESC guidelines for the diagnosis and management of chronic coronary syndromes. Eur Heart J. Published online 2020. doi:10.1093/eurheartj/ehz425

26. Mathews R, Wang W, Kaltenbach LA, et al.(2018) Hospital variation in adherence rates to secondary prevention medications and the implications on quality. Circulation. 137(20):2128-2138. doi:10.1161/CIRCULATIONAHA.117.029160

27. Schwalm JD, Ivers NM, Natarajan MK, et al.(2015) Cluster randomized controlled trial of Delayed Educational Reminders for Long-term Medication Adherence in ST-Elevation Myocardial Infarction (DERLA-STEMI). Am Heart J. 170(5):903-913. doi:10.1016/j.ahj.2015.08.014

28. Lansberg P, Lee A, Lee ZV, Subramaniam K, Setia S.(2018) Nonadherence to statins: Individualized intervention strategies outside the pill box. Vasc Health Risk Manag. 14:91-102. doi:10.2147/VHRM.S158641

29. van Driel ML, Morledge MD, Ulep R, Shaffer JP, Davies P, Deichmann R.(2016) Interventions to improve adherence to lipid-lowering medication. Cochrane Database Syst Rev. 2016(12). doi:10.1002/14651858.CD004371.pub4

30. Barankay I, Reese PP, Putt ME, et al.(2020) Effect of Patient Financial Incentives on Statin Adherence and Lipid Control. JAMA Netw Open. 3(10):e2019429. doi:10.1001/jamanetworkopen.2020.19429

31. De Backer G, Jankowski P, Kotseva K, et al.(2019) Management of dyslipidaemia in patients with coronary heart disease: Results from the ESC-EORP EUROASPIRE V survey in 27 countries. Atherosclerosis. 285:135-146. doi:10.1016/j.atherosclerosis.2019.03.014

32. Ray KK, Molemans B, Schoonen WM, et al.(2020) EU-Wide Cross-Sectional Observational Study of Lipid-Modifying Therapy Use in Secondary and Primary Care: the DA VINCI study. Eur J Prev Cardiol. Published online 2020:1-11. doi:10.1093/eurjpc/zwaa047

33. Coorey GM, Neubeck L, Mulley J, Redfern J.(2018) Effectiveness, acceptability and usefulness of mobile applications for cardiovascular disease self-management: Systematic review with metasynthesis of quantitative and qualitative data. Eur J Prev Cardiol. 25(5):505-521. doi:10.1177/2047487317750913

34. Thompson D, Mackay T, Matthews M, Edwards J, Peters NS, Connolly SB.(2017) Direct Adherence Measurement Using an Ingestible Sensor Compared With Self-Reporting in High-Risk Cardiovascular 
Disease Patients Who Knew They Were Being Measured: A Prospective Intervention. JMIR mHealth uHealth. 5(6):e76. doi:10.2196/mhealth.6998

35. Salzwedel A, Jensen K, Rauch B, et al.(2020) Effectiveness of comprehensive cardiac rehabilitation in coronary artery disease patients treated according to contemporary evidence based medicine: Update of the Cardiac Rehabilitation Outcome Study (CROS-II). Eur J Prev Cardiol. 27(16):1756-1774. doi:10.1177/2047487320905719

36. Giannuzzi P, Temporelli PL, Marchioli R, et al.(2008) Global secondary prevention strategies to limit event recurrence after myocardial infarction: Results of the GOSPEL study, a multicenter, randomized controlled trial from the Italian Cardiac Rehabilitation Network. Arch Intern Med. 168(20):2194-2204. doi:10.1001/archinte.168.20.2194

37. Simon-Tuval T, Neumann PJ, Greenberg D.(2016) Cost-effectiveness of adherence-enhancing interventions: A systematic review. Expert Rev Pharmacoeconomics Outcomes Res. 16(1):67-84. doi:10.1586/14737167.2016.1138858

38. Supervia M, Turk-Adawi K, Lopez-Jimenez F, et al.(2019) Nature of Cardiac Rehabilitation Around the Globe. EClinicalMedicine. 13:46-56. doi:10.1016/j.eclinm.2019.06.006

39. Ho PM, Bryson CL, Rumsfeld JS.(2009) Medication adherence: Its importance in cardiovascular outcomes. Circulation. 119(23):3028-3035. doi:10.1161/CIRCULATIONAHA.108.768986

40. Garber MC, Nau DP, Erickson SR, Aikens JE, Lawrence JB.(2004) The concordance of self-report with other measures of medication adherence: A summary of the literature. Med Care. 42(7):649-652. doi:10.1097/01.mlr.0000129496.05898.02

41. Lauffenburger JC, Fontanet CP, Isaac T, et al.(2020) Comparison of a new 3-item self-reported measure of adherence to medication with pharmacy claims data in patients with cardiometabolic disease. Am Heart J. 228:36-43. doi:10.1016/j.ahj.2020.06.012

\section{Declarations}

\section{Funding}

None of the authors received financial support for the research, authorship and/or publication of this article

\section{Conflict of interest/Competing interests}

Haralambos Karvounis declares lecture and advisory board honoraria from AstraZeneca, Boehringer Ingelheim, Genesis Pharma, Menarini, Novartis, Pfizer, Roche, and Servier. George Giannakoulas declares lecture and/or advisory board honoraria from Amgen, AstraZeneca, Elpen Pharmaceuticals, Menarini, MSD, Mylan, Novartis, Pfizer, Sanofi and Servier. Alexandra Arvanitaki is the recipient of the International Training and Research Fellowship EMAH Stiftung Karla Voellm (2020-2021), Krefeld, Germany. The rest of the authors declare no conflicts of interest.

\section{Ethics approval}

The study was approved by the local ethics committee and was carried out in accordance with the 
Declaration of Helsinki

\section{Consent to participate}

Informed consent was obtained from all individual participants included in the study

\section{Availability of data and code}

All the data and the code that have been used to the current study are available upon request to the corresponding author

\section{Authors' Contributions}

$S Z, I F, A B, V N, A V, E M, S Z, H K$, and GG contributed to the conception or design of the work. SZ, IF, AP, AK, $A B, D K, A T, E M, A A, K A$, and SM contributed to the acquisition, analysis, or interpretation of data for the work. SZ, IF, AK, and AA drafted the manuscript. AP, AB, DK, VN, HK, EM, SZ, GK, and GG critically revised the manuscript. All gave final approval and agreed to be accountable for all aspects of work, ensuring integrity and accuracy.

\section{Figures}
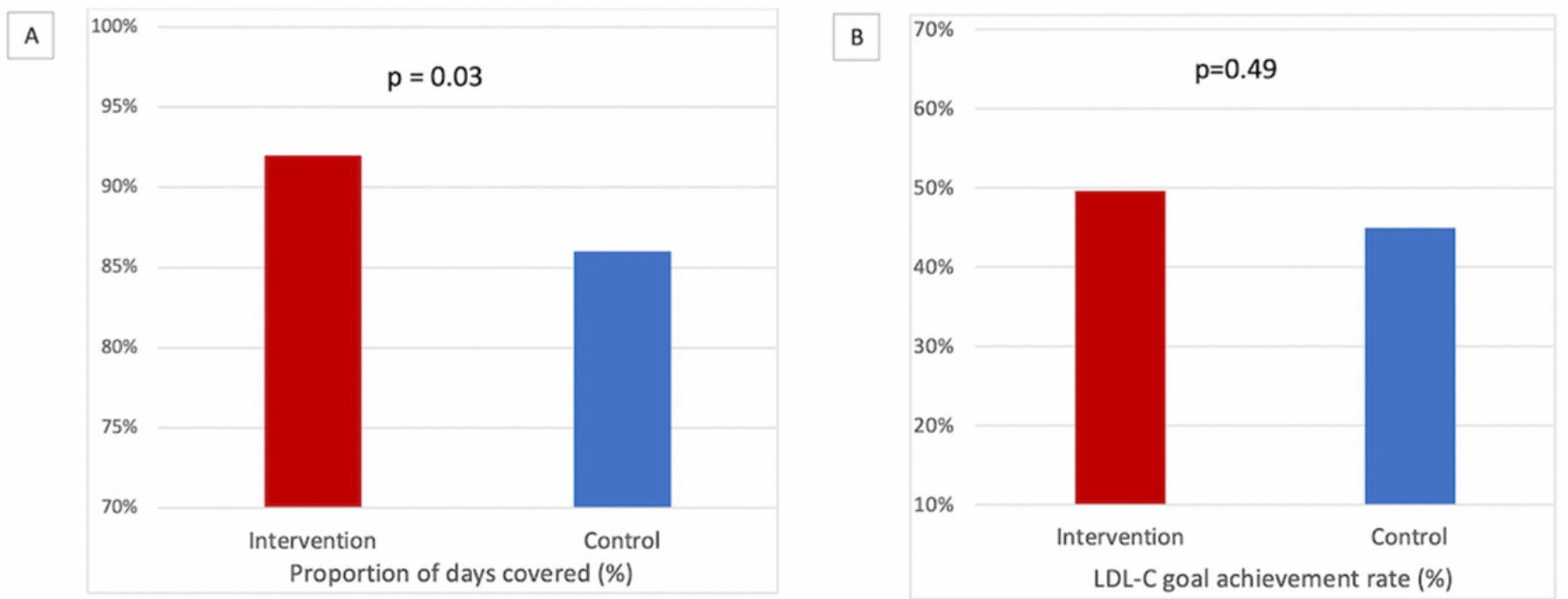

\section{Figure 1}

Primary outcomes Bar charts showing the adherence (proportion of days covered) (A) and LDL-C goal achievement (B) rates between the two experimental groups ( $p=0.03$ and $p=0.49$, respectively). 

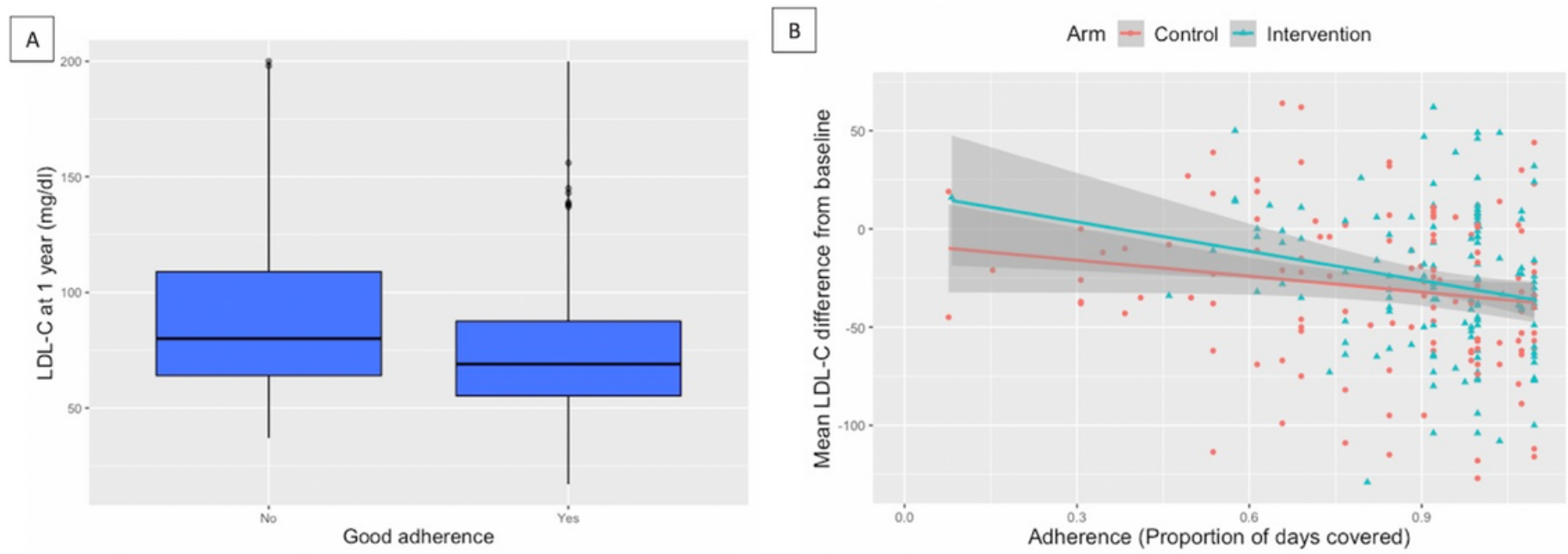

Figure 2

LDL-C and adherence (A) Boxplot showing the association between good adherence and LDL-C at 1-year $(p=0.004)$, (B) Scatterplot with regression line showing the change in LDL-C from baseline as a function of adherence over 1 year in the two experimental groups. The dots show observed data, and the lines indicate predicted values with $95 \%$ Cls for separate linear regressions of change in LDL-C on adherence alone.

A

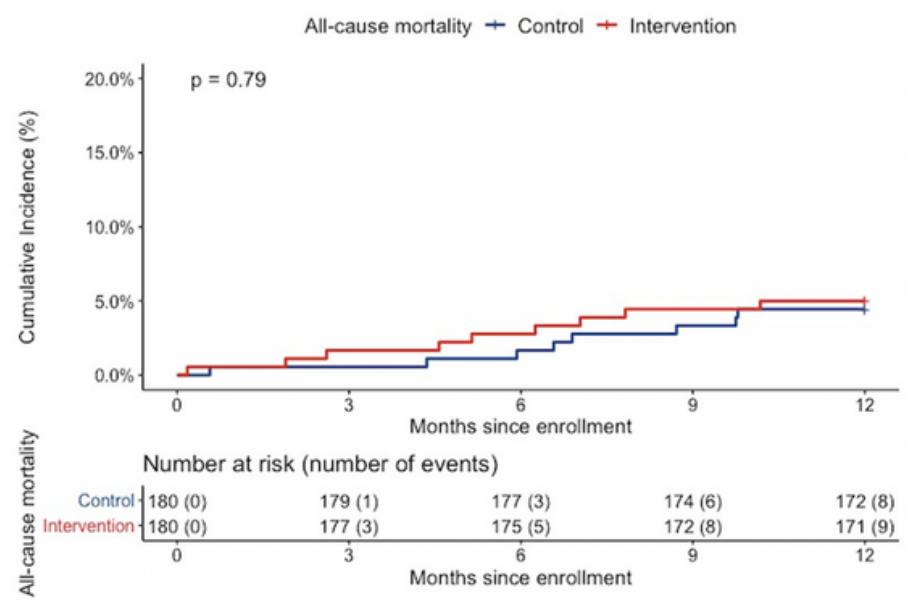

B

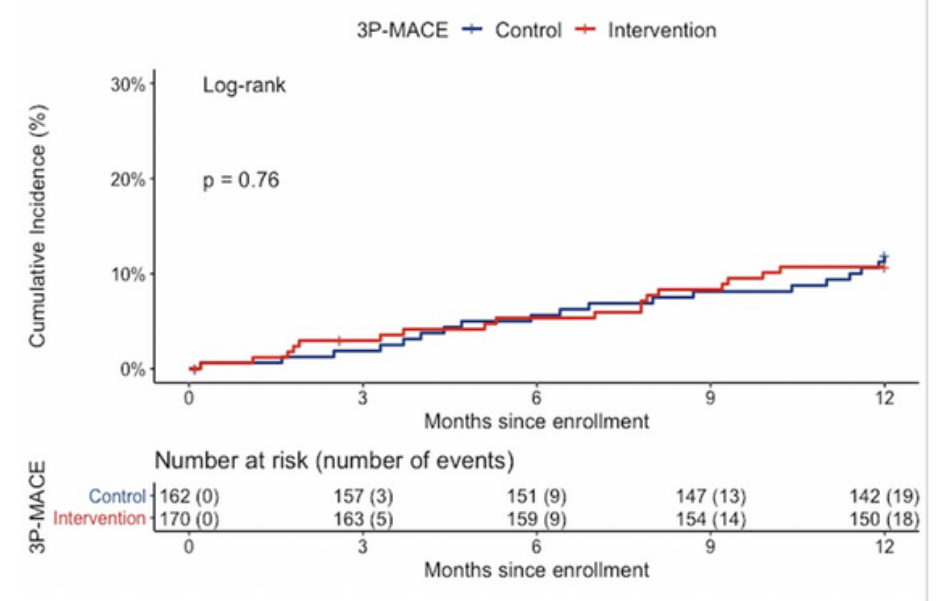

\section{Figure 3}

Clinical outcomes The cumulative incidences of the (A) death from any cause, (B) composite outcome, cardiovascular death, nonfatal myocardial infarction and nonfatal stroke were estimated with the use of the Kaplan-Meier method; hazard ratios and $95 \%$ confidence intervals were estimated with the use of Cox regression models, stratified according to treatment-group assignment as explanatory variables. 